medications such as liposomal amphotericin B, intravenous immunoglobulin therapy).

Pharmacological treatment included various diuretics, lowmolecular-weight heparin, correction of coagulation abnormalities, thrombocyte transfusions, albumin supplementation, ursodeoxycholic acid, acetylcysteine and defibrotide. Fluid balance was carefully monitored. The only patient who received defibrotide had faster clinical recovery and normalization of laboratory parameters. In three patients abdominal drainage and in two patients thoracic drainage had to be performed.

hepatic sinusoidal obstruction syndrome is a severe complication of oncologic treatment with possible lethal outcome requiring rapid diagnosis and intensive treatment.

\section{ADMINISTRATION OF TARGETED THERAPY IN CHILDREN WITH NTRK REARRANGED MESENCHYMAL NEOPLASMS}

A Bonevski*, J Stepan Giljevic, N Rajacic, I Topic, A Tripalo Batos, R Ivekovic, L Pazanin Department of hematology and oncology „Dr Mladen Cepulic', Children's Hospital Zagreb

\subsection{6/archdischild-2021-europaediatrics.308}

The aim of this report is to present 2 patients diagnosed with neoplasm of mesenchymal origin, in whom NTRK inhibitor entrectinib was succesfully administered.

The first patient presented at the age of 9 months as reccurent chalazion.

During subsequent clinical and radiological follow-up a diagnosis of right upper eyelid tumor was made. Taking into account tumor localization, surgical procedure of a maximum possible tumor reduction was performed, unfortunately with positive resection margins. Histopthological analysis confirmed the diagnosis of „NTRK-rearranged spindle cell neoplasm' according to the new 2020 WHO classification of soft tissue tumors. The neoplasm was charachterized by increased cellularity and relatively low mitotic activity. The possibility of an aggressive clinical course could not be excluded. Molecular analysis (FusionPlex Sarcoma Kit SAR6) verified an LMNANTRK fusion and oral entrectinib therapy was initiated. The patient has tolerated the medication very well and is without the radiological signs of eventual local relapse (magnetic resonance imaging).

The second patient is a 12-year-old boy presenting with a solid expansive lesion of the lesser pelvis extending towards the groin and anterior abdominal wall (locoregional disease). Tumor tissue sampling comfirmed malignant peripheral nerve sheath tumor. Neoadjuvant systemic chemotherapy according to the EpSSG-NRSTS protocol and radiotherapy accomplished only partial reduction of the tumor mass prompting next generation sequencing on formalin-fixed paraffinembedded tissue blocks (FoundationOne ${ }^{\circledR} \mathrm{Heme}$ ) that confirmed LMNA-NTRK1 fusion. Oral entrectinib has been initiated leading to tumor shrinkage that enabled complete surgical resection. The adverse effects registered in this case are one forearm fracture and increased appetite accompanied by significant weight gain that are diminished after medication dose reduction.

In conclusion, implementation of the targeted therapy has enabled avoidance of mutilating surgery and adverse effecets of conventional cytostatic agents in the first case. Excellent control of malignant mesenchymal tumor has been achieved in the second case.

\section{FANCONI ANEMIA: A CASE REPORT}

Alexandra M Rodrigues*, Joana Filipe Ribeiro, Catarina Francisco, Leonor Salicio. Hospital de Sousa Martins - Unidade Local Saúde da Guarda, Guarda, Portugal

\subsection{6/archdischild-2021-europaediatrics.309}

Background Fanconi anemia is a rare genetic inheritance disease. Although the transmission in most cases is autosomal recessive, in some instances, it can be X-linked. Congenital malformations are the first clinical presentation that could affect many organic systems like the skeletal, skin, cardiac, pulmonary, central nervous system, gastrointestinal and urogenital. Limb asymmetry, thenar hypoplasia, mycrocephaly and low stature and weight are very frequent. Patients may also present ear malformations with or without congenital deafness and intellectual disabilities. The most serious problem is early severe bone marrow failure which leads to pancytopenia and, some years later, to the progression of acute myeloid leukemia or myelodysplastic syndrome. Moreover, the presence of solid tumors (head and neck or anogenital) is more prevalent.

Case Report A 9 year old male child was born with polymalformations such as imperforate anus, micropenis, inverted nipples, low implanted ears, sacrococcigeal sinus, thenar and radius hypoplasia. His birth occurred under a supervised gestation of 42 weeks by caesarean because of fetal bradycardia. The Apgar index was 5/8/9 and reanimation was performed. The patient was hospitalized in intensive care and anus correction surgery was performed.

The child's parents were healthy but consanguineous (cousins). The boy is the couple's third child. The first one died at 7 years old with acute myeloid leukemia and had a previous diagnosis of Fanconi anemia. The second one had sudden death at 3 months old. Because of this, karyotype and evaluation of chromosomal breakage induced by diepoxybutane (DEB) was tested and the last one confirmed Fanconi anemia diagnosis. Until now, he developed pancytopenia and bone marrow failure without progression to leukemia. Later, he had a younger brother without Fanconi anemia. It should be noted that the child in question has a 17 year old male cousin also diagnosed with Fanconi anemia. The cousin has low stature, intellectual disability and performed Hematopoietic stem cell transplantation at 7 years old.

Currently, the child takes danazol and receives periodic transfusions. He has multidisciplinary follow-up and was proposed to Hematopoietic stem cell transplantation.

Conclusion Fanconi anemia is responsible for many malformations and inevitable bone marrow failure. The presence of an inheritance pattern increases the level of suspicion when other relatives have the disease.

After diagnosis, these patients need close multidisciplinary follow-up to improve the expectancy and quality of life. The prevention or early detection of neoplasia is the main objective for these patients, as it leads to poor prognosis.

\section{A GIRL WITH PRE-B ACUTE LYMPHOBLASTIC LEUKEMIA ON MAINTENANCE THERAPY WITH HEREDITARY COMPLEMENT-MEDIATED THROMBOTIC MICROANGIOPATHY (AHUS)}

Turudic Daniel*, Dejanovic-Bekic Sara, Mucavac Lucija, Pavlovic Maja, Milosevic Danko, Bilic Ernest. University Hospital Centre Zagreb

10.1136/archdischild-2021-europaediatrics.310 